\title{
Does Psychodrama Affect Perceived Stress, Anxiety-Depression Scores and Saliva Cortisol in Patients with Depression?
}

\author{
Lale Gönenir Erbay ${ }^{凶}$, İsmail Reyhani', Süheyla Ünal ${ }^{1}$, Cemal Özcan², \\ Tuba Özgöçer ${ }^{3}$, Cihat Uçar ${ }^{3}$, and Sedat Yıldız ${ }^{3}$ \\ ${ }^{1}$ Department of Psychiatry, Faculty of Medicine, Inonu University, Malatya, Turkey \\ ${ }^{2}$ Department of Neurology, Faculty of Medicine, Inonu University, Malatya, Turkey \\ ${ }^{3}$ Department of Physiology, Faculty of Medicine, Inonu University, Malatya, Turkey
}

\begin{abstract}
Objective Little is known about the effects of psychodrama group therapy on the level of stress, anxiety and depression of the group members, and the biological markers of stress. The aim of this study test cortisol level as a biological reflection of some mental characteristics gained by the psychodrama method in coping with stress.

Methods Depressive patients aged between 18 and 65 years, who diagnosed with depression according to DSM-5, without psychiatric comorbidity, who do not use drugs and were available for psychodrama were enrolled into the study. These patients were evaluated using the Perceived Stress Scale, State-Trait Anxiety Inventory and Beck Depression Inventory. In order to observe the circadian rhythm of cortisol, samples were collected from the participants before lunch, before dinner and before going to sleep on the day before psychodrama and on the 0,15 th, and 30th minutes after awakening in the morning of the psychodrama day; as well as just before and after the psychodrama session. Saliva cortisol level just before the initial session of psychodrama group therapy was compared with the saliva cortisol level just after the last psychodrama session at the end of 16 weeks.

Results Statistically significant difference was determined between the scores of perceived stress scale, STAI-1, STAI-2, beck depression inventory and salivary cortisol level before and after psychodrama session. There was significant decrease in both the scale scores and salivary cortisol after vs. before psychodrama.

Conclusion This results is important as it shows the biological aspect of clinical improvement. Further studies would provide us with better understanding of the effects of psychodrama group psychotherapy on depressive mood and biological projections by means of short-term and long-term follow-up studies.

Psychiatry Investig 2018;15(10):970-975
\end{abstract}

Key Words Psychodrama, Cortisol, Group therapy.

\section{INTRODUCTION}

Major depression, in which biological susceptibility plays an important role, is a mood disorder usually triggered by unfavorable life events. ${ }^{1}$ Conducting studies on the role of biological factors in the etiology of major depression has been possible with technological advancements. Abnormalities of the hypothalamic-pituitary-adrenal (HPA) axis are among

\footnotetext{
Received: March 27, 2018 Revised: July 3, 2018

Accepted: August 11, 2018

$\triangle$ Correspondence: Lale Gönenir Erbay, MD

Department of Psychiatry, Faculty of Medicine, Inonu University, 44280 Malatya, Turkey

Tel: +90-422-3410660-5405, Fax: +90-422-341-07-87,

E-mail: lalegonenir@hotmail.com

(a) This is an Open Access article distributed under the terms of the Creative Commons Attribution Non-Commercial License (http://creativecommons.org/licenses/by$\mathrm{nc} / 4.0$ ) which permits unrestricted non-commercial use, distribution, and reproduction in any medium, provided the original work is properly cited.
}

the predictors demonstrated most commonly and consistently. ${ }^{2}$ High cortisol level is determined in about $50 \%$ of depressive patients. ${ }^{3}$ Cortisol level increases in the hypothalamic-pituitary-adrenal (HPA) axis as a response to stress and plays a critical role in hemostasis. It is known that stress has a significant role in the initiation and maintenance of depression. It is also known that there are severe stressor agents prior to the depression attacks ${ }^{4}$ and that unpleasant experiences in early childhood enhance the risk of developing depression in the adulthood. ${ }^{5}$

Psychotherapeutic interventions are the methods known to have favorable impacts on the patients' coping with stress and on the quality of life and are recommended to be used particularly in depression. ${ }^{6,7}$ Group psychotherapy is a psychotherapeutic method performed with $8-10$ participants where the group rules such as respect, privacy and construc- 
tive feed-back, which in general help with establishing an environment of trust, are implemented. Proving the efficacy of group psychotherapies has always been the subject of interest for researchers. Early investigations have not confirmed the use of group therapies as an independent therapeutic method although they are auxiliary therapies. ${ }^{8}$ In the recent years, however, it has begun to be defined that group therapies have the ability of making objectively measurable changes in the patient's attitude, personality and behavior. ${ }^{9}$

Psychodrama is an experiential method of group therapy, which uses the role play technique in a managed way to make the participants to be aware of personal and interpersonal problems and work on the probable solutions via acting method, to see themselves from the outside, to try new roles, to gain different points of view and to change. It allows the group members to see the results of different behaviors and problem solution methods via intra group activities. These methods include expression of emotions and thoughts, prediction of personal and social skills, adaptation of new behaviors, and rehearsal for potential problems. ${ }^{10}$ Evidences indicating the efficacy of psychodrama group therapy are quite limited. In one of these limited studies, it was reported that drama-based interventions have significant impacts on self-conception and social functioning in the adolescents. ${ }^{11,12}$

Nevertheless, little is known about the effects of psychodrama group therapy on the level of perceived stress, anxiety and depression scores of the group members, and the biological markers of stress. This pilot study tested the hypothesis that cortisol level decreases as a biological reflection of some mental characteristics gained by the psychodrama method in coping with stress.

\section{METHODS}

Depressive patients aged between 18 and 65 years, who visited a psychiatry clinic of a university hospital, evaluated by a psychiatrist and diagnosed with depression according to DSM-5 [using The Structured Clinical Interview for DSM-5 (SCID-5)], without psychiatric comorbidity, who do not use drugs and were available for psychodrama and willing to join the group were enrolled into the study. Patients had no drug use and no psychiatric comorbidity. Whereas the psychodrama group consisted of 10 patients initially, the study was carried out with 8 patients since 2 patients thereafter left the group. Additional physical disorders, neurological and endocrine disorders, receiving drugs that may interfere with cortisol levels, and presence of additional psychiatric diagnoses that might be confounding factors in both group dynamics and cortisol levels were predetermined as exclusion criteria. Prior to the study, all patients were informed about the pro- cedure and their written consents were obtained (inönü university ethics committee, approval number:2013-204). They underwent minimal physical, neurological and psychiatric examination and laboratory testing.

Patients that agreed to participate in the study completed the following scales both prior to the psychotherapy process and after the group therapy sessions that lasted for 16 weeks.

Perceived Stress Scale (PSS) was developed by Cohen et al. ${ }^{13}$ PSS, which consisted of a total of 14 items, was designed to measure to what degree a set of situations are perceived as stressful. Participants rate each item over 5-point Likert scale ranging from "Never (0)" and "Very often (4)." Among these items, positive statements are rated reversely. The scores of PSS-changes between 0 and 40. High scores indicate higher level of perceived stress. Validity and reliability of the Turkish version was performed by Eskin et al. ${ }^{14}$

State-Trait Anxiety Inventory (STAI-I and STAI-II) was developed in 1983 by Spielberger et al. ${ }^{15}$ It was adapted into Turkish by Öner and Le Compte ${ }^{16}$ and validity and reliability study was done. Each of the subscales consists of 20 items. Answers are scored between 1 and 4. Total score obtained from each subscale ranges from 20 to 80 . High score indicates high anxiety level.

Beck Depression Inventory (BDI), which was developed by Beck and Steer ${ }^{17}$ and consists of 21 items, was used to measure depressive symptoms. Each item indicates a depressive symptom. BDI, which is a self-report tool, is the most common tool used to measure depression worldwide. Total score changes between 0 and 63 with higher scores indicating severer depression. In the validity and reliability study, coefficient of internal consistency reliability was calculated to be $0.84 .^{18}$

\section{Practice of psychodrama group psychotherapy}

Group practices were performed once in a week between 14:00 and 17:00 o'clock for 16 weeks in a place suitable for psychodrama group practice. Each session was managed by a therapist and a co-therapist under the supervision of a supervisor.

Analysis of salivary cortisol for cortisol levels
Cortisol level, which is one of the biological markers of
stress, is determined by means of the salivary cortisol level.
Saliva samples were collected according to the expert con-
sensus guidelines reported by Stalder et al. ${ }^{19}$ and following
the rules explained by Ozgocer et al. ${ }^{20}$ Experimental proce-
dure and study protocol were briefly explained to the partici-
pants during face-to-face interview. Polypropylene tubes (1.5
mL, ISOLAB Laborgeräte GmbH, Wertheim, Germany) la-
beled with the participant's ID and sample number (e.g. S1, 
S2, S3, S0, S15, S30, S4, and S5) were given to the participants. Passive drool method was used to collect saliva samples. Participants were warned to avoid any kind of activities such as eating, drinking, and brushing teeth in the first 30 min after waking up and before the collection of other samples. Drinking water was allowed, provided that it has been stopped at least $10 \mathrm{~min}$ before the next saliva sampling. Cortisol secretion shows a circadian rhythm; it peaks in the morning and then gradually declines during day time. An other aspect of cortisol secretion is called as cortisol awakening response (CAR). This acuterise, which is superposed on the normal cycle, reaches peak level about $30 \mathrm{~min}$ after awakening. ${ }^{21}$ Attention was paid for the samples to be collected from all participants at the same hour, during fasting and after resting. In order to observe the circadian rhythm of cortisol, samples were collected from the participants before lunch, before dinner and before going to sleep on the day before psychodrama and on the 0,15 th, and 30th minutes after awakening in the morning of the psychodrama day; as well as just before and after the psychodrama session. Study design is illustrated in Figure 1.

However, as the number of saliva samples collected from the participants is not high enough to perform the statistical analyses, after session vs. before session comparison could not be performed. Saliva cortisol level just before the initial session of psychodrama group therapy was compared with the saliva cortisol level just after the last psychodrama session at the end of 16 weeks.

\section{Saliva cortisol analysis}

Salivary samples were analyzed for cortisol level by enzyme-linked immunosorbent assay (ELISA) developed in our laboratory. ${ }^{22}$ In brief, cortisol-BSA stock solution was diluted with carbonate buffer and used at an amount of $200 \mu \mathrm{L} /$ well in a 96-well microtiter plate. The plate was incubated overnight at $+4 \mathrm{C}$ and then washed with wash buffer. After blocking the unbound sites, standard solutions or samples were added in triplicate ( $40 \mu \mathrm{L} /$ well). Addition of specific

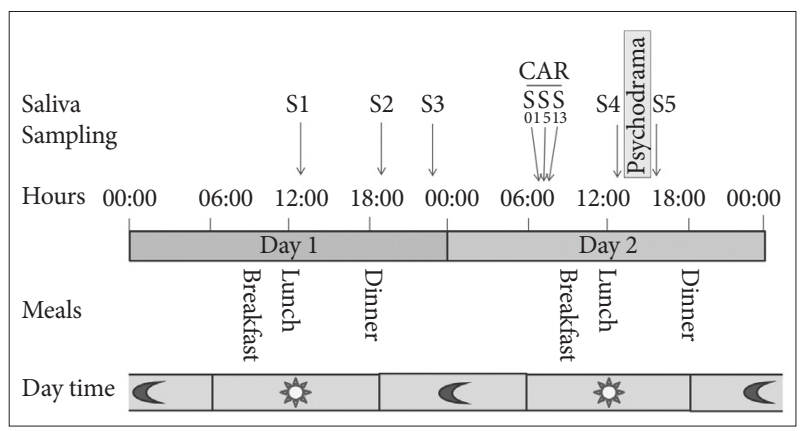

Figure 1. Study design. CAR: cortisol awakening response, S: sample number. primary antibody ( $40 \mu \mathrm{L} /$ well) was followed by addition of biotinylated secondary anti-rabbit antibody $(100 \mu \mathrm{L} /$ well $)$. Incubations were carried out at $37^{\circ} \mathrm{C}$ for $45 \mathrm{~min}$ and at $37^{\circ} \mathrm{C}$ for $30 \mathrm{~min}$, respectively for the last two stages. At the end of each stage, the plate was washed and finally streptavidin peroxidase solution $(100 \mu \mathrm{L} /$ well $)$ was added and the plate was incubated at $37^{\circ} \mathrm{C}$ for $15 \mathrm{~min}$. The plate was washed again and tetramethylbenzidine substrate $(150 \mu \mathrm{L} /$ well $)$ was added, and then it was incubated in the dark medium for 10 min. After the addition of stop solution (sulfuric acid $10 \%$, $50 \mu \mathrm{L} /$ well), the absorbance was measured at $450 \mathrm{~nm}$ using a plate reader spectrophotometer (BioTek, Synergy HT, Winooski, VT, USA). Cortisol concentrations were quantified using the program in the spectrophotometer. ${ }^{22}$

\section{Statistical analysis}

Statistical analyses were performed by the Statistical Program for Social Sciences-SPSS for Windows, version 17.0 (SPSS Inc., Chicago, IL, USA). Descriptive statistics were presented as mean and standard deviation for numerical variables and as number and percentage for categorical variables. Nonparametric test was used to compare the numerical variables in casecondition for normal distribution was not met. A $p$ value $<0.05$ was considered statistically significant.

\section{RESULTS}

The psychodrama group consisted of 8 subjects, of which 4 were male and 4 were female. The mean age was $34.37 \pm 11.75$ years. None of the participants had physical comorbidity. All of them were being followed for depression, and the mean follow-up period was $5.37 \pm 7.02$ years. None of the participants were consuming alcohol or smoking. Other sociodemographic characteristics of the group are demonstrated in Table 1.

The mean diurnal salivary cortisol level of depressive patients participating in the 16-week psychodrama sessions are illustrated in Figure 2.

Correlation between diurnal salivary cortisol levels is illustrated in Figure 3.

Statistically significant difference was determined between the scores of perceived stress scale, STAI-1, STAI-2, beck depression inventory and salivary cortisol level before and after psychodrama session. There was significant decrease in both the scale scores and salivary cortisol after vs. before psychodrama (Table 2).

\section{DISCUSSION}

The primary objective of the present study was to investi- 


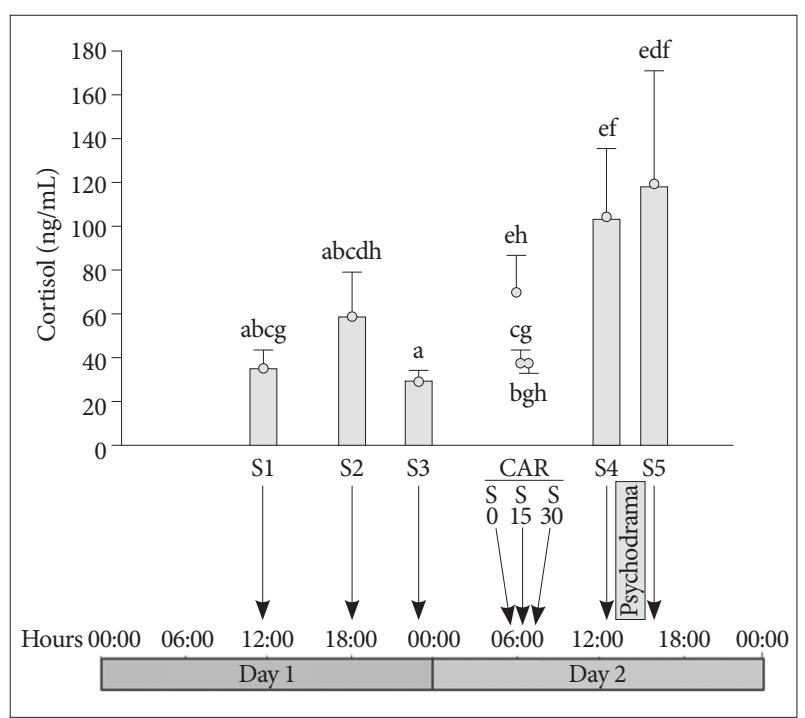

Figure 2. Mean diurnal salivary cortisol levels in depressive patients participated in the weekly psychodrama sessions for 16 weeks. Saliva samples were collected in two consecutive days every 4 weeks until the end of the study. Cortisol values represent the mean of entire study period. The S1-S3 denotes saliva sampling for the assessment of diurnal cortisol release; S4-S5 denotes saliva sampling before and after the psychotherapy session in the next day; S0, S15 and S30 denotes saliva sampling for cortisol awakening response (CAR) just after awakening (SO) and 15 (S15) and 30 (S30) min after awakening. Columns (or points) with different letters differ significantly by paired t-test. In summary, cortisol levels were the lowest in the midnight sample (S3) but the highest just before and after the group psychotherapy session. a: before lunch, one day before psychodrama, b: before diner, one day before psychodrama, c: before sleeping, one day before psychodrama, d: 0 minutes after waking in the morning, e: 15 minutes after waking in the morning, f: 30 minutes after waking in the morning, g: before psychodrama, h: after psychodrama.

gate the effect of psychodrama group therapy on the level of perceived stress, level of state-trait anxiety, Beck depression scores and accordingly salivary cortisol levels in depressive patients.

It is known that both biological and psychological factors play a role in the development of depression. The relationship between cortisol over secretion and depression is one of the oldest investigations in the field of biological psychiatry. Primarily the activation of HPA axis and increased glucocorticoid activity attract attention when the organism faces with stress. It is known that diurnal rhythm of cortisol is impaired in the subjects suffering from depression, or changes in cortisol level may lead to depressive symptoms. ${ }^{23}$ Even though plasma cortisol levels are frequently measured in depressive patients, it is observed that the cortisol peaks in these patients do not increase in number compared to normals. For this reason, depressed cortisol hypersecretion is not due to the peak value of plasma cortisol, but is due to excess secretion on each peak. ${ }^{24}$ Symptoms that accompany depression such as sleep disorders, decreased libido, cardiovascular changes and cognitive dysfunction indicate that HPA axis as

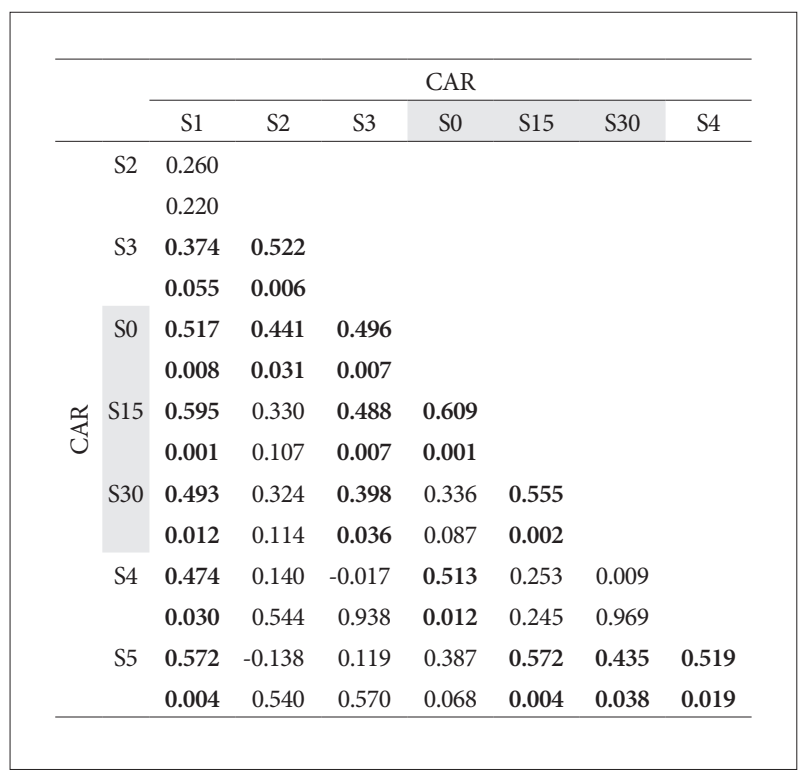

Figure 3. Pearson correlations among the log10-converted salivary cortisol data. In each cell, the upper value is the correlation coefficient $(r)$ and the lower value is the $p$ value. Significant correlations are shown in bold. In general, all data seem to be inter-correlated, but S1 cortisol levels (sample taken after the lunch on Day 1) best describe the data taken afterwards (except S2). Additionally, S3 level (sample at midnight) is positively and significantly correlated with all cortisol awakening response (CAR) data. Data before and after psychodrama session (S4 and S5, respectively) are also positively correlated.

well is involved in the development of depression. Plasma cortisol concentration is increased, metabolites of cortisol are increased, and 24-hour urinary free cortisol concentration is increased in the majority of patients with major depression. ${ }^{25}$ Increased cortisol production during depression returns to the normal levels with resolution of depression; i.e., it appears to be a state marker ${ }^{26}$ Moreover, also the normal circadian rhythm of cortisol is impaired in depressive patients in addition to the increased basal cortisol levels. HPA axis changes are more prominent in the patients with severer depression and in older patients. ${ }^{27}$ There is a very limited number of studies examining the effect of psychotherapies on cortisol levels. One of these studies examined the effect of SSRI and psychotherapy on cortisol levels in generalized anxiety disorder. Older adults with GAD who received both escitalopram and CBT demonstrated a significant reduction in peak cortisol levels at posttreatment compared to the group who received escitalopram without CBT augmentation. ${ }^{28}$ Psychodrama group psychotherapy is a method used for the treatment of depression. It was determined that depressive symptoms are improved and symptom severity is decreased after treatment with psychodrama. ${ }^{29}$ Huntley et al. ${ }^{30}$ conducted a systematic review and meta-analysis to investigate the efficacy of group-based psychological therapy in depression and concluded that psychotherapies except for CBT 
Table 1. Sociodemographic and some clinical characteristics of the psychodrama group

\begin{tabular}{|c|c|}
\hline & $\mathrm{N}(\%)$ \\
\hline \multicolumn{2}{|l|}{ Gender } \\
\hline Male & $4(50)$ \\
\hline Female & $4(50)$ \\
\hline \multicolumn{2}{|l|}{ Marital status } \\
\hline Married & $3(37.5)$ \\
\hline Single & $5(62.5)$ \\
\hline \multicolumn{2}{|l|}{ Number of children } \\
\hline None & $5(62.5)$ \\
\hline 1 & $1(12.5)$ \\
\hline$\geq 2$ & $2(25)$ \\
\hline \multicolumn{2}{|l|}{ Residential area } \\
\hline Urban & $3(37.5)$ \\
\hline Rural & $5(62.5)$ \\
\hline \multicolumn{2}{|l|}{ Education status } \\
\hline High school & $3(37.5)$ \\
\hline College/University & $5(62.5)$ \\
\hline \multicolumn{2}{|l|}{ Household } \\
\hline Spouse and/or children & $3(37.5)$ \\
\hline Parents & $5(62.5)$ \\
\hline \multicolumn{2}{|l|}{ Income (TL) } \\
\hline$\leq 500$ & $1(12.5)$ \\
\hline $500-1000$ & $3(37.5)$ \\
\hline $1000-2000$ & $2(25)$ \\
\hline$\geq 2000$ & $2(25)$ \\
\hline \multicolumn{2}{|l|}{ Occupation } \\
\hline Yes & $1(12.5)$ \\
\hline No & $7(87.5)$ \\
\hline \multicolumn{2}{|c|}{ Social support other than the family } \\
\hline Yes & $5(62.5)$ \\
\hline No & $3(37.5)$ \\
\hline \multicolumn{2}{|l|}{ Stress in the last month } \\
\hline None & $2(25)$ \\
\hline Occupational problems & $1(12.5)$ \\
\hline Financial problems & $2(25)$ \\
\hline Living conditions & $1(12.5)$ \\
\hline Illness of family members & $2(25)$ \\
\hline \multicolumn{2}{|l|}{ Stress in the last 6 months } \\
\hline None & $1(12.5)$ \\
\hline Occupational problems & $1(12.5)$ \\
\hline Financial problems & $2(25)$ \\
\hline Living conditions & $1(12.5)$ \\
\hline Illness of family members & $3(37.5)$ \\
\hline
\end{tabular}

Table 2. Change in the scale scores and salivary cortisol level after vs. before psychodrama

\begin{tabular}{lccc}
\hline & $\begin{array}{c}\text { Before } \\
\text { psychodrama } \\
(\text { mean } \pm \text { SD })\end{array}$ & $\begin{array}{c}\text { After } \\
\text { psychodrama } \\
(\text { mean } \pm \text { SD })\end{array}$ & p value \\
\hline $\begin{array}{l}\text { Perceived } \\
\text { stress scale }\end{array}$ & $19.37 \pm 5.50$ & $7.37 \pm 2.44$ & 0.011 \\
STAI- 1 & $45.87 \pm 8.20$ & $27.12 \pm 4.32$ & 0.011 \\
STAI-2 & $52.87 \pm 7.95$ & $34.12 \pm 5.08$ & 0.012 \\
Beck depression & $21.37 \pm 11.24$ & $3.37 \pm 1.59$ & 0.012 \\
$\quad$ inventory & & & \\
Salivary cortisol & $76.97 \pm 86.16$ & $18.50 \pm 4.08$ & 0.014 \\
\hline SD: standard deviation, STAI: State-Trait Anxiety Inventory
\end{tabular}

have limited efficacy. Despite the outcomes that psychodrama group psychotherapy contributes to the decrease in depressive symptoms and increase in the subjects' social adaptation, ${ }^{31}$ studies on this field are limited in number. Moreover, biological projection of clinical improvement as well has not been studied enough. It is reported that psychotherapies, which have different theories and methods of implementation known to be effective in the treatment of depression, have improving effect as in drug therapy on the abnormalities that occur with depression in the metabolism and activity of prefrontal cortex. ${ }^{32}$ The present study aimed to test the hypothesis that psychodrama group therapy is clinically effective and that the changes in a biological marker like salivary cortisol accompany the improvement.

The results of the present study reveal statistically significant decrease in the level of perceived stress, state-trait anxiety scores and severity of depression with psychodrama group psychotherapy. Decrease in the same direction was determined also in the salivary cortisol level. This is important as it shows the biological aspect of clinical improvement.

The limitations of the present study include limited patient number, evaluation of only the patients with mild-moderate depression, and measurement of neurochemical abnormalities in depression only by means of salivary cortisol level.

Further studies would provide us with better understanding of the effects of psychodrama group psychotherapy on depressive mood and biological projections by means of short-term and long-term follow-up studies.

\section{REFERENCES}

1. Kendler KS, Karkowiski LM, Prescott CA. Causal relationship between stresful life events and the onset of depression. AMJ Psychiatry 1999;156:837-841.

2. Lopez JF, Akil H, Watson SJ. Neural circuits mediating stress. Biol Psychiatry 1999;46:1461-1471.

3. Kartalc1 Ş, Eşel E, Özsoy S, Kula M, Turan T. Neurosteroids and cognitive functions in depressed women. Bull Clin Psychopharmacol 
2008;18:9-21.

4. Checkley S. Neuroendocrine mechanisms and the precipitation of depression by life events. Br J Psychiatry 1992;160:7-17.

5. Weiss El, Longhurst JG, Mazure CM. Childhood sexual abuse as a risk factor for depression in women: psychosocial and neurobiological correlates. Am J Psychiatry 1999;156:816-828.

6. Fortune DG, Richards HL, Griffiths CE, Main CJ. Targeting cognitivebehavior therapy to patients' implicit model of psoriasis: results froma patientpreference controlled trial. Br J Clin Psychol 2004;43:65-82.

7. Varma GS, Karadağ F, Oğuzhanoğlu NK, Özdel O. The role of group psychotherapy and psychodrama in the treatment of depression. Klinik Psikiyatri 2017;20:308-317.

8. Stotsky B, Zolik E. Group psychotherapy with psychotics: 1921-1963: a review. Int J Group Psychother 1965;15:321-344.

9. Taylor NT, Burlingame GM, Fuhriman A, Kristensen KB, Johansen J, Dahl D. A survey of mental health care provider and managed care organization attitudes toward, familiarity with, and use of group interventions. Int J Group Psychother 2001;51:243-263.

10. Azoulay B, Orkibi H. The four-phase CBN psychodrama model: a manualized approach for practice and research. Art Psychother 2015;42:10-18

11. Tsiaras A. Dramatic play as a means of developing primary school students' self-concept. J Drama Theatre Educ Asia 2012;3:47-67.

12. Guli LA, Semrud-Clikeman M, Lerner MD, Britton N. Social Competence Intervention Program (SCIP): a pilot study of a creative drama program for youth with social difficulties. Art Psychother 2013;40:37-44.

13. Cohen S, Kamarck T, Mermelstein R. A global measure of perceived stress. J Health Soc Behav 1983;24:385-396.

14. Eskin M, Harlak H, Demirkıran F, Dereboy Ç. Adaptation of Perceived Stress Scale to Turkish: Reliability and Validity Analysis. Yeni Sempozyum Dergisi 2013;51:132-140.

15. Spielberger CD, Gorsuch R.L, Lushene R, Vagg PR, Jacobs GA. Manual for the State-Trait Anxiety Inventory. Palo Alto, CA: Consulting Psychologists Press; 1983.

16. Öner N, Le Compte A. State-Trait Anxiety Inventory Handbook. İstanbul: Boğaziçi University Publications; 1983.

17. Beck AT, Steer RA. Beck Depression Inventory Manual. San Antonio, TX: The Psychological Corporation; 1987.

18. Hisli N. A study on the validity of the Beck Depression Inventory. Türk Psikoloji Dergisi 1989;7:3-13.

19. Stalder T, Kirschbaum C, Kudielka BM, Adam EK, Pruessner JC, Wüst
S, et al. Assessment of the cortisol awakening response: expert consensus guidelines. Psychoneuroendocrinology 2016;63:414-432.

20. Ozgocer T, Yildiz S, Uçar C. Development and validation of an enzyme-linked immunosorbent assay for detection of cortisol in human saliva. J Immunoassay Immunochem 2017;38:147-164.

21. Steptoe A, Cropley M, Griffith J, Kirschbaum C. Job strain and anger expression predict early morning elevations in salivary cortisol. Psychosom Med 2000;62:286-292.

22. Ozgocer T, Ucar C, Yildiz S. Cortisol awakening response is blunted and pain perception is increased during menses in cyclic women. Psychoneuroendocrinology 2017;77:158-164.

23. Swaab DF, Bao AM, Lucassen PJ. The stress system in the human brain in depression and neurodegeneration. Ageing Res Rev 2005;4:141-194.

24. Nogay A. Cortisol secretion and serotonin in depressive patients. Klinik Piskofarmakoloji Bülteni 1991;1:30-33.

25. Albayrak EÖ, Ceylan ME. Neurobiological factors in etiology of depression. Düşünen Adam 2004;17:27-33

26. Musselman DL, Neineroff CB. Depression and endocrine disorders: focus on the thyroid and adrenal systein. Br J Psychiatry 1996;168:123-128.

27. Beck-Friis J, Ljunggren JG, Thorén M, von Rosen D, Kjellman BF, Wetterberg L. Melatonin, cortisol, and ACTH in patients with inajor depressive disorder and healthy hum.an with special reference to the outcome of the dexamethasone suppression test. Psychoneuroendocrinology 1985;10:173-186.

28. Rosnick CB, Wetherell JL, White KS, Andreescu C, Dixon D, Lenze EJ. Cognitive-behavioral therapy augmentation of SSRI reduces cortisol levels in older adults with generalized anxiety disorder: a randomized clinical trial. J Consult Clin Psychol 2016;84:345-352.

29. Gatta M, Lara DZ, Lara DC, Andrea S, Paolo TC, Giovanni C. Analytical psychodrama with adolescents suffering from psycho-behavioral disorder: Short-term effects on psychiatric symptoms. Art Psychother 2010;37:240-247.

30. Huntley AL, Araya R, Salisbury C. Group psychological therapies for depression in the community: systematic review and meta-analysis. $\mathrm{Br}$ J Psychiatry 2012;200:184-190.

31. Hamamc1 Z. Integrating psychodrama and cognitive behavioral therapy to treat moderate depression. Art Psychother 2006;33:199-207.

32. Goldapple K, Segal Z, Garson C, Lau M, Bieling P, Kennedy S, et al. Modulation of cortical-limbic pathways in major depression: treatment-specific effects of cognitive behavior therapy. Arch Gen Psychiatry 2004;61:34-41. 